\title{
The COVID-19 Pandemic: Role of Supportive Care in Cancer Patients
}

\author{
Wasimul Hoda ${ }^{1}$, Sachidanand Jee Bharati², Abvinav Kumar ${ }^{3}$, Sanjay Kumar ${ }^{1}$, \\ Chandrashekhar Choudhari ${ }^{4}$, Abhishek Shankar ${ }^{5}$
}

${ }^{1}$ Rajendra Institute of Medical Sciences, India. ${ }^{2}$ Oncoanaesthesia \& palliative Medicine, All India Institute of Medical Sciences, India. ${ }^{3}$ All India Institute of Medical Sciences, India. ${ }^{4}$ Rajiv Ratan Central Hospital, India. ${ }^{5}$ Lady Hardinge Medical College \& SSK Hospital, India.

\begin{abstract}
Cancer care is adversely affected by the corona virus disease 2019 (COVID-19). Due to increasing number of COVID-19 cases, all healthcare resources are diverted to take care of COIVD-19 patients. This is the most difficult time for cancer patients because cancer treatment is getting delayed or interrupted due to COVID-19. In this tough time, the supportive care can be continued with basic minimum resources and can provide a big relief to cancer patients.
\end{abstract}

Keywords: Supportive care- triage- psychosocial support- end of life

\section{Introduction}

A pandemic brings enormous sufferings through illness, anxiety, stress and death with inevitable financial $\&$ social instability. The response to such situation needs taking care of all the sufferings related to it. Cancer patients are even more vulnerable due the immunological and psychosocial impact of malignancy and its ongoing therapy [1]. Here comes the major role of supportive care in the form of palliation. Palliation is an ethical obligation even at the time of pandemic. In the context of current COVID-19 pandemic, delivery and continuity of palliative care for patients with cancer within resource constrained health systems is a concern and a challenge.

\section{Challenges in Cancer Patients}

Cancer being a time sensitive disease and patients appear to be at increased risk of COVID-19 infection. The prime concern and challenge is related to patient safety. The dilemma revolves around, whether cancer patients should visit hospital to continue their treatment as coming out of homes can expose them to COVID infection. Also, cancer treatment can compromise their immunity putting them at increasing risk of infection.
The dilemma continues because the available evidence is limited. According to initial data, the outcomes are worse than individuals without cancer [2]. Older age groups and patients with systemic diseases are prone to have more severe COVID-19 symptoms and bad outcomes [3].

Lack of trained palliative care physicians and availability of morphine and other essential medications at their native places are another big challenges which forces cancer patients to visit hospitals and exposing themselves to infections. The institutional based challenges, such as focus of care and diversion of facilities to COVID patients, neglecting the cancer patients resulting in deviation from treatment protocols and leading to fear, anxiety and sufferings.

The overall impact of this pandemic on cancer patients is significant as it is going to affect the management, quality of life and survival [4]. Priority based cancer treatment plans are being decided on the basis of risk/ benefit ratio. In conditions where the risk of Severe Acute Respiratory Symptom Corona Virus (SARS CoV 2) infection outweighs the expected cancer related outcome, shared decision making while discussing the potential

Corresponding Author:

Dr. Sachidanand Jee Bharati

Oncoanaesthesia \& Palliative Medicine, All India Institute of Medical Sciences, India.

Email: Sachidadr@Yahoo.Co.In 
risks and benefits of planned treatment, will empower patients and caregivers to prioritize their preferences [5].

\section{Need of Supportive Care in Cancer Patients}

Supportive care focuses on improving quality of life (QOL) of patients and their family members. Cancer patient needs more support in form of palliative care since the day of diagnosis. The need for care increases at the time of pandemic when there are enormous uncertainty, stress and dilemma. The current situation has halted the smooth delivery of care to cancer patients. Therefore there is need of a comprehensive care plan based on holistic care approach.

The major domains identified from evidence available globally in guiding health care workers can be summarized as TRI-SPEED approach for cancer patients. It helps in providing holistic care combating current pandemic situation.

TRI-SPEED approach (Figure 1):

\section{TRI- Triage}

2. S- Symptom Control

3. P- Psychosocial Support

4. E- Ethical based care discussion

5. ED- End of life Care

\section{Triage}

Priority based cancer therapies in the context of COVID pandemic is the need of hour. It can be done by Triaging patients based on symptom burden which helps in prioritizing care and leads to proper utilization of resources [6-7]. Taking care of limited resources, the decision to either continue or stop cancer therapy should be made before going for aggressive treatments. Irrespective of condition of cancer patients the palliative care services should continue till end in the form of telecommunication, follow up, home based care and bereavement if possible. Triage towards most urgent palliative care cases with severe uncontrolled symptoms like pain, oncological emergencies such as spinal cord compression should be done and treated.

Triaging system for patients on priority basis with advanced cancer who do not have COVID-19 disease is summarized in Figure 2. Symptom management and psychosocial support should continue throughout the process at each step of management. There will be some patients with advanced and metastatic cancer, and some may be in older age group. They all constitute a group with low priority. Deferring treatment in such patients

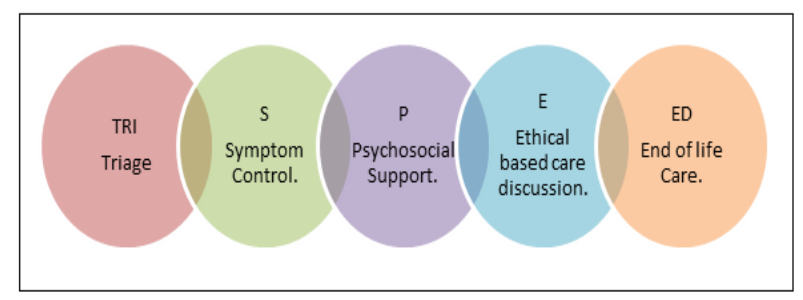

Figure 1. TRI-SPEED Approach for Providing Supportive Care in Cancer Patients

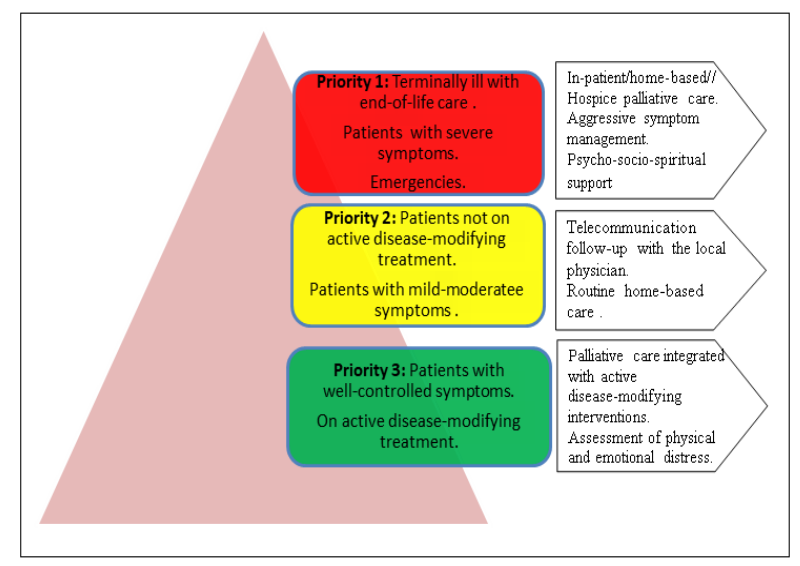

Figure 2. Triaging System for Patients in non- COVID Cancer Patients [8].

will bring a fresh wave of uncertainty, fear, and physical suffering.

\section{Symptom Control}

Patients with advanced cancers present with both physical and psychosocial symptoms. Acknowledgement and adherence to key palliative care principles is needed for adequate symptom management and care. There is a need of careful attention to minimize suffering including end of life care. The common symptoms like pain, nausea and vomiting can be managed at home. Extra morphine tablets can be given to patients in order to prevent frequent visits to hospital. The home based palliative care worker plays a vital role in it. For patients with severe dyspnea, sepsis and pneumonia, it must be differentiated with COVID-19. Therefore, suspected patients should be screened for COVID 19 and managed as per the existing guidelines. Use of telecommunication helps in managing symptoms at home and allows a hospital free follow up.

\section{Psychosocial Support}

This Pandemic has also brought a surge of fear, uncertainty, anxiety and depression. The magnitude is even more in cancer patients, survivors and their family members. The patients who are not able to continue their cancer therapy due to restricted movements in lockdown are filled with guilt and depressive thoughts. There is a strong need of psychosocial support to such patients. Empathetic communication, honesty, trust, compassion with patient and support to family throughout the process are important components in such cases. The emerged psychological crisis needs evidence based management, training of ground level health care workers in communication skills, use of telecommunication and home based palliative care [9].

\section{Ethical Based Care Discussion}

In view of limited resource and shifting of focus more towards COVID patients, the decision making process in cancer patients for and against an intervention creates a major ethical challenge. Effective communication and detailed discussion with patient, family, primary physician 
and palliative care team can simplify the process. Documentation of decision is an important part of ethical based discussion. The patients who are denied of further medical intervention shouldn't feel neglected. This is possible with sensitive and compassionate discussion with patient and family members.

\section{End of life Care}

In addition to care and symptom management, communication and compassion matters in patients with advanced cancer. Use of technology like telecommunication, recorded messages, music and prayers can be advocated. Patients at terminal stages with refractory symptoms can be managed with palliative sedation. The impact of the COVID-19 pandemic on end-of-life care must be addressed as it may not be possible at the family's preferred place. There may be limitations on the presence of family members at funeral process due to government policies. Realistic hope and true communication regarding the prognosis is the key. This leads to a confidence and trust among the caregivers and doctors who are engaged to provide better bereavement process.

In conclusion, video conferencing and telemedicine practice, triaging cancer patients on priority basis, minimizing risk/benefit ratio for interventions with palliative intent and acknowledging the importance of spiritual care are crucial in overcoming the challenges posed by current pandemic in cancer patients. The comprehensive care can be achieved by the proposed TRI-SPEED approach.

\section{References}

1. Pietrantonio F, Garassino MC. Caring for Patients With Cancer During the COVID-19 Outbreak in Italy. JAMA Oncology. 202006 01;6(6):821. https://doi.org/10.1001/ jamaoncol.2020.1426

2. Liang W, Guan W, Chen R, Wang W, Li J, Xu K, Li C, Ai Q, Lu W, Liang H, Li S, He J. Cancer patients in SARSCoV-2 infection: a nationwide analysis in China. The Lancet Oncology. 2020 03;21(3):335-337. https://doi.org/10.1016/ s1470-2045(20)30096-6

3. Zhou F, Yu T, Du R, Fan G, Liu Y, Liu Z, Xiang J, Wang Y, Song B, Gu X, Guan L, Wei Y, Li H, Wu X, Xu J, Tu S, Zhang Y, Chen H, Cao B. Clinical course and risk factors for mortality of adult inpatients with COVID-19 in Wuhan, China: a retrospective cohort study. The Lancet. 2020 03;395(10229):1054-1062. https://doi.org/10.1016/s01406736(20)30566-3

4. Shankar A, Saini D, Roy S, Mosavi Jarrahi A, Chakraborty A, Bharti S et al. Cancer Care Delivery Challenges Amidst Coronavirus Disease - 19 (COVID-19) Outbreak: Specific Precautions for Cancer Patients and Cancer Care Providers to Prevent Spread. Asian Pacific Journal of Cancer Prevention. 2020;21(3):569-573. https://doi.org/10.31557/ apjcp.2020.21.3.569

5. Mohanti B. Ethics in Palliative Care. Indian Journal of Palliative Care. 2009;15(2):89. https://doi.org/10.4103/09731075.58450

6. Hanna TP, Evans GA, Booth CM. Cancer, COVID-19 and the precautionary principle: prioritizing treatment during a global pandemic. Nature Reviews Clinical Oncology.
202004 02;17(5):268-270. https://doi.org/10.1038/s41571020-0362-6

7. Shankar A, Seth T, Saini D, Bharati S, Roy S. Oncology Nursing Challenges during COVID-19 Outbreak: Precautions and Guidance. Asia-Pacific Journal of Oncology Nursing. 2020;7(4):305-307. https://doi.org/10.4103/apjon. apjon_39_20

8. Rao K, Singhai P, Rao S, Salins N. Palliative care for advanced cancer patients in the COVID-19 pandemic: Challenges and adaptations. Cancer Research, Statistics, and Treatment. 2020;3(5):127. https://doi.org/10.4103/crst.crst_130_20

9. Rajkumar RP. COVID-19 and mental health: A review of the existing literature. Asian Journal of Psychiatry. 2020 08;52:102066. https://doi.org/10.1016/j.ajp.2020.102066

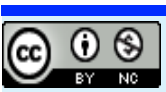

This work is licensed under a Creative Commons AttributionNon Commercial 4.0 International License. 